\title{
Title: Supramolecular secondary ion mass spectrometry - revealing molecular surroundings and inter-molecular interactions in organic matter
}

\author{
Authors: M. Kawecki ${ }^{1,2}$, L. Bernard ${ }^{1}$, D. Passerone ${ }^{3}$, R. Crockett $^{1}$
}

\author{
Affiliations: \\ ${ }^{1}$ Laboratory for Surface Science \& Coating Technologies, Empa, 8600 Dübendorf, Switzerland. \\ ${ }^{2}$ Department of Physics, University of Basel, 4056 Basel, Switzerland. \\ ${ }^{3}$ Nanotech @ Surfaces laboratory, Empa, 8600 Dübendorf, Switzerland. \\ *Correspondence to: m.kawecki.wenger@gmail.com
}

\section{Abstract:}

While supramolecular chemistry is a firmly established research field in laboratory conditions, the experimental study of non-covalent interactions, such as hydrogen bonding and piinteractions, between different molecules in chemically rich, non-crystalline matter remains highly challenging. We demonstrate that soft ion bombardment can trigger the joint desorption of weakly interacting adjacent molecules, and that this in turn allows molecular interaction probabilities, surroundings, and arrangement in organic matter to be probed. Assemblies of organic molecules linked by hydrogen bonds or dipole- $\pi$ interactions are extracted here with preservation of chemistry and structure as single-charged supramolecular secondary ions. Among the examples shown is the desorption of stable 12-molecular clusters of the amino acid L-proline, which suggest an icosahedral on-surface self-assembly, and the desorption of supramolecular linear oligomers of 2,5-piperazinedione. The second half of the study lays down the statistical framework for the reconstruction of a molecular interactome based on the relative abundances of supramolecular dimers of different compositions within a mass spectrum acquired on organic matter containing more than one type of molecule.

One Sentence Summary: The collective extraction of groups of adjacent molecules as singlecharged supramolecular secondary ions provides a window into molecular interactions, arrangement and surroundings in complex organic systems.

\section{Introduction}

For over 30 years, mass spectrometry has provided a unique tool to probe molecular interactions and supramolecular structures $(1,2)$. Prominently, electrospray ionization (ESI), was successfully used in the study of non-covalent ligand-receptor $(3)$ and protein-protein $(4,5)$ interactions, and its potential has recently been expanded to the study of complex membrane protein assemblies (6). To date, however, the mass spectrometric study of molecular interactions and supramolecular structures is limited to liquid (3-6), or solution processed (7) sample material. Hard ionization techniques, such as secondary ion mass spectrometry (SIMS), which allow solid matter to be studied, result in pronounced molecular fragmentation and are commonly considered too energetic to preserve multi-molecular ions. Contrary to this common perception, as early as 1979 supramolecular secondary ions detected from solid nitrogen $\left(\mathrm{N}_{2}\right)$ probed at the temperature of $15 \mathrm{~K}$ were observed, and clusters up to $\left[\mathrm{N}_{2}\right]_{22}{ }^{+}$were reported (8). 
Soon after, ion impact-induced chemical reactions leading to complicated cluster chemistries were reported for solid $\mathrm{N}_{2} \mathrm{O}, \mathrm{NO}, \mathrm{N}_{2} \mathrm{O}_{3}$ and $\mathrm{N}_{2} \mathrm{O}_{4}$ probed with heavy monoatomic primary ions, which led to the conclusion that the composition of extracted clusters need not be indicative of the composition of the solid (9). From today's perspective, these latter observations seem to have discouraged any potential further research into the extraction of intact supramolecular clusters by means of SIMS. Meanwhile, instrumentation evolved and in particular cluster primary ion sources emerged $(10,11)$ which allow much softer probing of the surface in SIMS.

We demonstrate here that intact supramolecular assemblies can be routinely extracted. We show that the mass spectrometric analysis of the fraction of extracted supramolecular clusters that possesses charge provides insight into molecular self-assembly at the singlenanometer scale. This is exemplified by revealing the on-surface self-assembly of the amino acid L-proline into stable, closed 12-molecular structures. We proceed by demonstrating that also subtle changes in the supramolecular structuring of complex organic matter can be probed. This is exemplified by the documentation of enhanced alignment of the small molecule 2,5piperazinedione into linear supramolecular polymers in the presence of dextran. We conclude by showing that the compositional distribution of extracted supramolecular assemblies can be also used to estimate probabilities for the occurrence of non-covalent bonding between specific molecules.

The samples studied consist of drop-deposited films of organic molecules, dried on oxygen plasma-treated silicon wafers. The drop-deposited films of several micro-meters thickness are probed at ambient temperature using $\mathrm{Bi}_{3}{ }^{+}$primary ions in a fully commercial instrument.

\section{Results and Discussion}

\section{Supramolecular secondary ions and their relation to the supramolecular structures in a system probed}

Analyzing the high-mass range of mass spectra we initially acquired for SIMS reference spectra databases of all proteinogenic amino acids and a set of 19 monosaccharides (12-15), we observe that each single spectrum contains peaks that can be attributed to supramolecular ions, consisting of multiple molecules held together by hydrogen bonding. A positive time-of-flight (ToF) SIMS spectrum acquired on a drop-deposited film of L-proline is shown in Fig. 1A. The strong peak at mass $116.07 \mathrm{u}$ corresponds to unfragmented proline molecules, ionized through acceptance of a positive hydrogen ion. Further major peaks occur at masses corresponding to $[\mathrm{nM}+\mathrm{H}]^{+}$, where $\mathrm{n}$ is an integer larger than $1, \mathrm{M}$ the molecular mass of proline and $\mathrm{H}$ the mass of hydrogen. In the negative spectrum, a similar pattern is observed, with strong intensities for masses that correspond to $[\mathrm{nM}-\mathrm{H}]^{-}$(Fig. 1B). We explicitly observe $[\mathrm{nM} \pm \mathrm{H}]^{ \pm}$patterns in the amino-acid and monosaccharide spectra, as opposed to, for example, [nM-(n-1) $\left.\left(\mathrm{H}_{2} \mathrm{O}\right) \pm \mathrm{H}\right]^{ \pm}$or $\left[\mathrm{nM}-(\mathrm{n}-1) \mathrm{H}_{2} \pm \mathrm{H}\right]^{ \pm}$patterns which would emerge from chemical reactions to form covalent bonds. Therefore, the monomer units of the secondary ions consisting of $n$ molecules, such as can be seen for L-proline in Fig. 1A, are held together by non-covalent attractive interactions. In amino acids, the strongest non-covalent interactions occur through hydrogen bonds. Other non-covalent interactions are also observed to lead to $[\mathrm{nM}+\mathrm{H}]^{+}$-type peaks. For example, despite 2-(2naphthyl)ethylamine not forming hydrogen bonds with itself, a high-intensity $[2 \mathrm{M}+\mathrm{H}]^{+}$peak is 
detected in the positive polarity spectra acquired on pure drop-deposited 2-(2naphthyl)ethylamine (Fig. 1C). We conducted quantum chemistry simulations using the rangeseparated Meta-GGA functional M11 (16) with full structural optimization, and found that the most stable conformation of two 2-(2-naphthyl)ethylamine molecules is through dipole- $\pi$ noncovalent bonding (Fig. 1C, inset), resulting in an interaction strength of $-0.40 \mathrm{eV}$. The benchmark for detectable non-covalent interactions is yet lower and was set by the ToF-SIMS measurements conducted in 1979 on $\mathrm{N}_{2}$ condensed on a copper substrate cooled to a temperature of $15 \mathrm{~K}$, where positively charged cluster secondary ions of $\mathrm{N}_{2}$ were reported (8). Quantum chemistry simulations conducted by us estimate the binding energy, dominated by London forces, between two $\mathrm{N}_{2}$ molecules to be approximately $-0.02 \mathrm{eV}$.
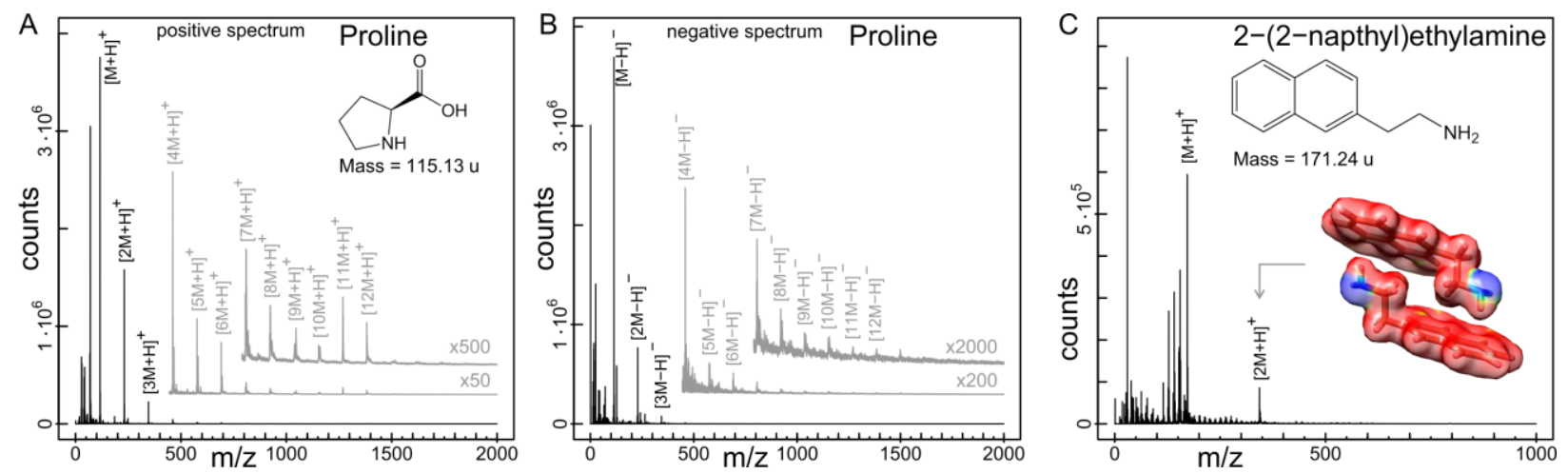

Fig. 1. Supramolecular secondary ions in ToF-SIMS mass spectra. (A) ToF-SIMS positive polarity spectrum of pure L-proline drop-deposited on a silicon wafer. (B) ToF-SIMS negative polarity spectrum of pure L-proline. The spectrum was acquired on the same sample as the positive polarity spectrum presented in Fig. 1A. (C) ToF-SIMS positive polarity spectrum of pure 2-(2-napthyl)ethylamine. The modeled conformation of the supramolecular dimer is depicted as inset, showing a density isosurface at 0.02 a.u. colored with the electrostatic potential, blue being negative (min. value: $-0.02 E_{h} / e$ ); red positive (max. value: $0.025 E_{h} / e$ ).

The $[\mathrm{nM}+\mathrm{H}]^{+}$peaks in all proteinogenic amino acids and many more organic molecules, not only those capable of hydrogen bonding, are a strong indication that the extraction of supramolecular secondary ions is not an exception but a common phenomenon, when cluster primary ions are used (see also Supplementary Materials, Figs. S1-S5). The coexistence of intact supramolecular assemblies and strongly fragmented species within a single mass spectrum can be explained by the supramolecular secondary ions presumably desorbing further away from the primary ion impact location than the routinely studied fragment secondary ions. In contrast to early studies conducted using heavy mono-atomic primary ions (9), we observe here that the intensity of peaks assigned to potential chemical reaction products among the secondary ions remains several orders of magnitude below those of intact supramolecular clusters extracted as such from the bulk. In the positive proline spectrum (Fig. 1A), the increased intensity of the $[11 \mathrm{M}+\mathrm{H}]^{+}$and $[12 \mathrm{M}+\mathrm{H}]^{+}$peaks, and the absence of supramolecular secondary ions with $\mathrm{n}>12$, indicate that the drop-deposited proline arranges into closed icosahedral clusters of 12 proline molecules each. This molecular arrangement has been previously observed to form during the liquid-gas transition of evaporating micro-droplets (17). Note the particularly strong intensity of the $[11 \mathrm{M}+\mathrm{H}]^{+}$secondary ion in Fig. 1A which is in line with the positive ionization of a closed 12-proline cluster by loss of a single $[\mathrm{M}-\mathrm{H}]^{-}$molecular ion. 
In order to provide an example of how supramolecular secondary ions can be used to assess supramolecular structuring in complex organic matter, measurements on drop-deposited samples containing 2,5-piperazinedione were carried out (Fig. 2). 2,5-piperazinedione is capable of forming double hydrogen-bonds at two opposing sites, and is known from X-ray diffraction to form ribbons of linear supramolecular polymers upon crystallization $(18,19)$. A positive polarity mass spectrum acquired on pure drop-deposited 2,5-piperazinedione is shown in Fig. 2A. Figure 2B shows the positive polarity spectrum of a drop-deposited film consisting of dextran $\left(\mathrm{M}_{\mathrm{n}}=5^{\prime} 000\right)$ and 2,5-piperazinedione in a mass ratio of 5:1. Despite the dilution of the 2,5-piperazinedione, the high intensity peaks in the positive mass spectrum are still dominated by the characteristic $[\mathrm{nM}+\mathrm{H}]^{+}$pattern observed in Fig. 2A. However, with the dextran present (Fig. 2B), the intensity ratios of the successive $[\mathrm{nM}+\mathrm{H}]^{+}$2,5-piperazinedione peaks normalized to the monomer unit peak $\left([\mathrm{M}+\mathrm{H}]^{+}\right)$are $1: 0.82: 0.019: 0.0047: 0.00082$ for $n \in\{1: 5\}$, whereas on pure 2,5-piperazinedione (Fig. 2A) these intensity ratios are $1: 0.55: 0.015: 0.0038: 0.00062$. The increase in normalized $[\mathrm{nM}+\mathrm{H}]^{+}$peak intensities indicates that the presence of dextran reinforces the alignment of 2,5-piperazinedione into supramolecular linear polymers. We propose that the difference in hydration layer around the dextran coils and the 2,5-piperazinedione molecules makes these two systems mutually exclusive, an effect similar to that observed in aqueous biphasic systems $(20,21)$.

Additionally, we observe that beyond a threshold primary ion dose density limit, the intensities of the 2,5-piperazinedione $[\mathrm{nM}+\mathrm{H}]^{+}$peaks rapidly drop below the level of noise (see Fig. 2C). The vanishing of $[\mathrm{nM}+\mathrm{H}]^{+}$peaks with accumulated ion impact damage confirms that the supramolecular secondary ions do not form in a process of rearrangements induced by the primary ion impact, but are directly related to the supramolecular structure of the bulk. Note, only measurements acquired at primary ion dose densities below the degradation onset are compared in the other figures in this study.
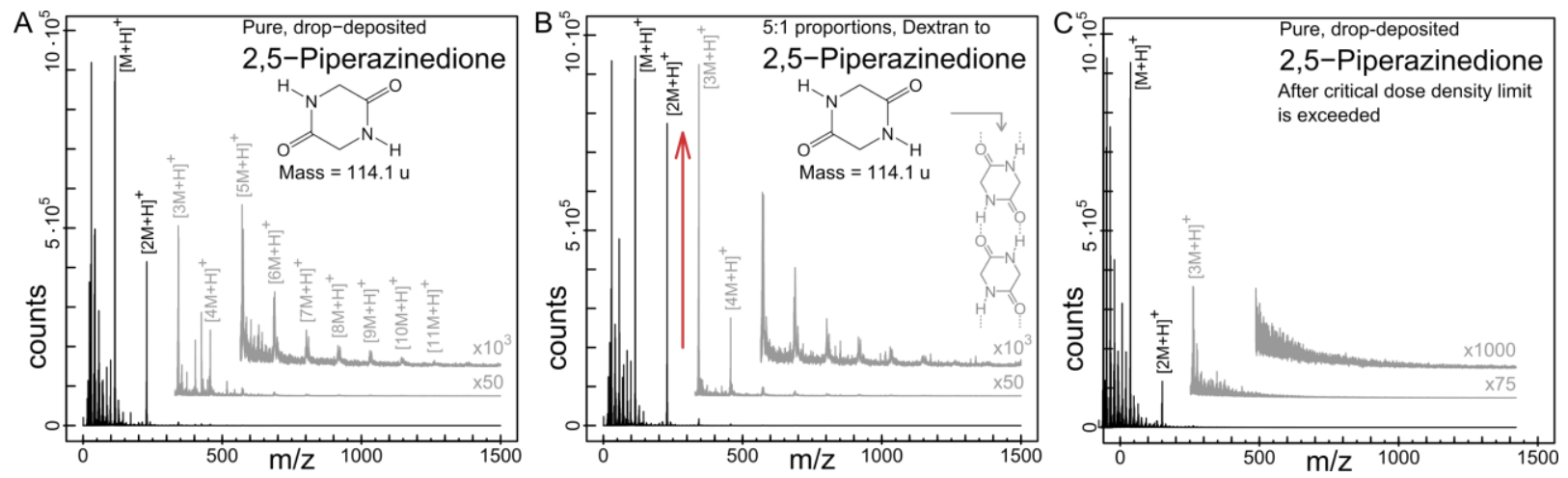

Fig. 2. Supramolecular structuring of 2,5-piperazinedione in a dextran matrix. (A) ToFSIMS positive polarity spectrum of pure 2,5-piperazinedione. (B) Positive polarity spectrum of 2,5-piperazinedione in dextran $\left(\mathrm{M}_{\mathrm{n}}=5^{\prime} 000\right)$ in the total mass ratio 1:5. (C) Positive polarity spectrum of pure 2,5-piperazinedione summing the last 10 scans (scans 91-100) of a spectrum acquired on the same sample as panel $A$. The average accumulated dose density for each scan here is $8.0 * 10^{12}$ ions $/ \mathrm{cm}^{2}$. The average accumulated ion dose density for the scans in panel A is $4.6 * 10^{11}$ ions $/ \mathrm{cm}^{2}$. Both measurements sum over a total of 10 scans, but the acquisition in panel A suffered no prior ion impact damage. 


\section{A mathematical framework for probing molecular interaction probabilities in systems consisting of multiple different molecules}

The extraction and detection of assemblies consisting of weakly bound molecules can provide insight into the chemical surroundings of a selected molecule in a complex organic environment. Information on the probability that a molecule $\mathrm{M}_{\mathrm{i}}$ and a molecule $\mathrm{M}_{\mathrm{j}}$ will noncovalently interact is contained in the $\left[\mathrm{M}_{\mathrm{i}}+\mathrm{M}_{\mathrm{j}}+\mathrm{H}\right]^{+}$ion count. Assuming that the extraction $\left(\mathrm{p}_{\mathrm{ij}}^{\text {ext }}\right)$, ionization $\left(\mathrm{p}_{\mathrm{ij}}^{\mathrm{ion}}\right)$, transmission $\left(\mathrm{p}_{\mathrm{ij}}^{\text {tra }}\right)$ and detection $\left(\mathrm{p}_{\mathrm{ij}}^{\text {det }}\right)$ probabilities for a weakly bound pair $\left(\mathrm{M}_{\mathrm{i}}+\mathrm{M}_{\mathrm{j}}\right)$ were known, the number of such $\left(\mathrm{M}_{\mathrm{i}}+\mathrm{M}_{\mathrm{j}}\right)$ pairs on the surface probed can be related to the the $\left[\mathrm{M}_{\mathrm{i}}+\mathrm{M}_{\mathrm{j}}+\mathrm{H}\right]^{+}$counts as follows:

$$
\mathrm{n}_{\mathrm{ij}}=\alpha^{-1} \cdot \frac{\left[\mathrm{M}_{\mathrm{i}}+\mathrm{M}_{\mathrm{j}}+\mathrm{H}\right]^{+}}{\mathrm{p}_{\mathrm{ij}}^{\mathrm{ext}} \cdot \mathrm{p}_{\mathrm{ij}}^{\mathrm{ion}} \cdot \mathrm{p}_{\mathrm{ij}}^{\mathrm{tra}} \cdot \mathrm{p}_{\mathrm{ij}}^{\mathrm{det}}}
$$

Here, $n_{i j}=: n\left(M_{i}+M_{j}\right)$ is the total number of $\left(M_{i}+M_{j}\right)$ pairs within the area probed, and $\alpha$ is the surface fraction affected by primary ion impact.

First a system that consists of only two types of molecules, $\mathrm{M}_{1}$ and $\mathrm{M}_{2}$ shall be considered. In this binary system, a molecule of the type $M_{1}$ can either interact with a molecule of the type $M_{2}$, or with a different $\mathrm{M}_{1}$ molecule. The fraction $\mathrm{X}_{12}^{1}$ of $\mathrm{M}_{1}$ molecules that, out of these two possible options, interacts with an $\mathrm{M}_{2}$ molecule can be expressed by the following equation:

$$
\begin{aligned}
& X_{12}^{1}= \frac{n\left(M_{1}+M_{2}\right)}{n\left(M_{1}+M_{2}\right)+n\left(M_{1}+M_{1}\right)} \\
&= \frac{\left[M_{1}+M_{2}+H\right]^{+}}{p_{12}^{\text {ext }} \cdot p_{12}^{\text {ion }} \cdot p_{12}^{\text {tra }} \cdot p_{12}^{\text {det }}} \\
& \frac{\left[M_{1}+M_{2}+H\right]^{+}}{p_{12}^{\text {ext }} \cdot p_{12}^{\text {ion }} \cdot p_{12}^{\text {tra }} \cdot p_{12}^{\text {det }}}+\frac{\left[2 M_{1}+H\right]^{+}}{p_{11}^{\text {ext }} \cdot p_{11}^{\text {ion }} \cdot p_{11}^{\text {tra }} \cdot p_{11}^{\text {det }}}
\end{aligned}
$$

In the nomenclature chosen, the superscript in $\mathrm{X}_{12}^{1}$ denotes the molecule whose interactions are of interest (here $\mathrm{M}_{1}$ ) and the subscript denotes the two specific interaction partners, one of which is the molecule denoted in the superscript. The secondary ion counts for $\left[\mathrm{M}_{1}+\mathrm{M}_{2}+\mathrm{H}\right]^{+}$and $\left[\mathrm{M}_{1}+\mathrm{M}_{1}+\mathrm{H}\right]^{+}$are acquired in one single spectrum. (Note, the value of $\mathrm{X}_{12}^{1}$ is independent of the surface fraction affected by primary ion impact, $\alpha$.) A priori, extraction, ionization, transmission and detection probabilities are not known. In a first approximation, the extraction, transmission, and detection probabilities are considered equal for all supramolecular dimers of comparable mass and bond strength. Equation 2 then becomes: 


$$
\mathrm{X}_{12}^{1} \approx \frac{\frac{\left[\mathrm{M}_{1}+\mathrm{M}_{2}+\mathrm{H}\right]^{+}}{\mathrm{p}_{12}^{\text {ion }}}}{\frac{\left[\mathrm{M}_{1}+\mathrm{M}_{2}+\mathrm{H}\right]^{+}}{\mathrm{p}_{12}^{\text {ion }}}+\frac{\left[2 \mathrm{M}_{1}+\mathrm{H}\right]^{+}}{\mathrm{p}_{11}^{\text {ion }}}}
$$

The ionization probabilities are, however, considered to depend on the supramolecular pairing. The ionization probability of an extracted $\left(\mathrm{M}_{\mathrm{i}}+\mathrm{M}_{\mathrm{j}}\right)$ pair can be estimated from the ion count of its individual molecules as:

$$
\begin{aligned}
\mathrm{p}_{\mathrm{ij}}^{\text {ion }} & \approx \mathrm{p}_{\mathrm{i}}^{\text {ion }}+\mathrm{p}_{\mathrm{j}}^{\text {ion }} \\
& =\frac{\left[\mathrm{M}_{\mathrm{i}}+\mathrm{H}\right]^{+}}{\mathrm{c}_{\mathrm{i}} \cdot \mathrm{p}_{\mathrm{i}}^{\text {ext }} \cdot \mathrm{p}_{\mathrm{i}}^{\text {tra }} \cdot \mathrm{p}_{\mathrm{i}}^{\text {det }}}+\frac{\left[\mathrm{M}_{\mathrm{j}}+\mathrm{H}\right]^{+}}{\mathrm{c}_{\mathrm{j}} \cdot \mathrm{p}_{\mathrm{j}}^{\text {ext }} \cdot \mathrm{p}_{\mathrm{j}}^{\text {tra }} \cdot \mathrm{p}_{\mathrm{j}}^{\text {det }}}
\end{aligned}
$$

where $c_{i, j}$ are the molecular concentrations on the sample surface and must be known or determined by a suitable experimental method. Using Equation 4, Equation 3 becomes:

$$
\begin{aligned}
& \mathrm{X}_{12}^{1} \approx
\end{aligned}
$$

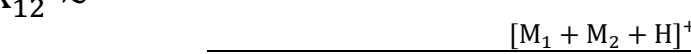

$$
\begin{aligned}
& \frac{\left[M_{1}+M_{2}+H\right]^{+}}{\left[M_{1}+H\right]^{+} /\left(c_{1} \cdot p_{1}^{\text {ext }} \cdot p_{1}^{\text {tra }} \cdot p_{1}^{\text {det }}\right)+\left[M_{2}+H\right]^{+} /\left(c_{2} \cdot p_{2}^{\text {ext }} \cdot p_{2}^{\text {tra }} \cdot p_{2}^{\text {det }}\right)}+\frac{\left[2 M_{1}+H\right]^{+}}{2\left[M_{1}+H\right]^{+} /\left(c_{1} \cdot p_{1}^{\text {ext }} \cdot p_{1}^{\text {tra }} \cdot p_{1}^{\text {det }}\right)}
\end{aligned}
$$

Considering the extraction, transmission and detection probabilities equal for molecules of comparable mass, analogue to the approximation made before for supramolecular dimers, Equation 5 is reduced to:

$$
\mathrm{X}_{12}^{1} \approx \frac{\frac{\left[\mathrm{M}_{1}+\mathrm{M}_{2}+\mathrm{H}\right]^{+}}{\left[\mathrm{M}_{1}+\mathrm{H}\right]^{+} / \mathrm{c}_{1}+\left[\mathrm{M}_{2}+\mathrm{H}\right]^{+} / \mathrm{c}_{2}}}{\frac{\left[\mathrm{M}_{1}+\mathrm{M}_{2}+\mathrm{H}\right]^{+}}{\left[\mathrm{M}_{1}+\mathrm{H}\right]^{+} / \mathrm{c}_{1}+\left[\mathrm{M}_{2}+\mathrm{H}\right]^{+} / \mathrm{c}_{2}}+\frac{\left[2 \mathrm{M}_{1}+\mathrm{H}\right]^{+}}{2\left[\mathrm{M}_{1}+\mathrm{H}\right]^{+} / \mathrm{c}_{1}}}
$$

If now in the two-molecular model system the concentrations of $\mathrm{M}_{1}$ and $\mathrm{M}_{2}$ are known, Equation 6 can be used to estimate the fraction of molecules $M_{1}$ that interact with a molecule $M_{2}$ and the fraction of molecules $M_{1}$ that interact with its own kind. Extending Equation 6 to a system consisting of $n$ molecules of comparable masses results in:

$$
\mathrm{X}_{\mathrm{ij}}^{\mathrm{i}} \approx \frac{\frac{\left[\mathrm{M}_{\mathrm{i}}+\mathrm{M}_{\mathrm{j}}+\mathrm{H}\right]^{+}}{\left[\mathrm{M}_{\mathrm{i}}+\mathrm{H}\right]^{+} / \mathrm{c}_{\mathrm{i}}+\left[\mathrm{M}_{\mathrm{j}}+\mathrm{H}\right]^{+} / \mathrm{c}_{\mathrm{j}}}}{\sum_{n} \frac{\left[\mathrm{M}_{\mathrm{i}}+\mathrm{M}_{n}+\mathrm{H}\right]^{+}}{\left[\mathrm{M}_{\mathrm{i}}+\mathrm{H}\right]^{+} / \mathrm{c}_{\mathrm{i}}+\left[\mathrm{M}_{n}+\mathrm{H}\right]^{+} / \mathrm{c}_{n}}}
$$


In the framework of the assumptions made, equations of the type (7) can be used to estimate the interaction partner distribution for a molecule of interest $\mathrm{M}_{\mathrm{i}}$ by analysis of its supramolecular dimer secondary ions.

In order to investigate this approach towards the determination of molecular interaction partner distributions, a model system consisting of the small molecules glycine, asparagine and 2,5piperazinedione in the molar ratio 1:1:1, drop deposited as a film on clean, plasma-treated silicon, was used. All three of these molecules interact through hydrogen bonds. The positive spectrum acquired on the above system is plotted in Fig. 3. The intensities of the peaks of interest (see Fig. 3) are shown in Table 1. For each of the three molecules in the drop-deposited film consisting of glycine (Gly), asparagine (Asp) and 2,5-piperazinedione (2,5-P.) in the molar ratio $1: 1: 1$, the interaction partner distribution (expressed in percent) is shown in Table 2 as calculated for different normalization assumptions (panels A-D). Panels A and C display the ineraction partner distributions as calculated making no assumption on dimer ionization probability, while in panels B and D Equation 7 is used. Simultaneously, in panels C and $\mathrm{D}$ it is taken into account that the $[\mathrm{Gly}+\mathrm{Asp}+\mathrm{H}]^{+}$dimer peak is below the noise floor as defined by the intensity of the surrounding $1 \mathrm{u}$ periodic peaks and its value is set to zero. In panels $\mathrm{A}$ and $\mathrm{B}$, the value of $[\mathrm{Gly}+\mathrm{Asp}+\mathrm{H}]^{+}$is left untouched. The data shown in Table 2 is plotted in Fig 4. The bar height indicates the interaction partner distributions as calculated by means of Equation 7 (Table 2, panel D). The error bars indicate the range of values obtained based on the different assumptions made as shown in Table 2 (panels A-D). Based on the normalized $\left[\mathrm{M}_{\mathrm{i}}+\mathrm{M}_{\mathrm{j}}+\mathrm{H}\right]^{+}$supramolecular dimer intensities, the qualitative conclusion is that each of the three molecules has a strong preference to associate with its own kind. This conclusion is independent of the specific normalization assumptions.

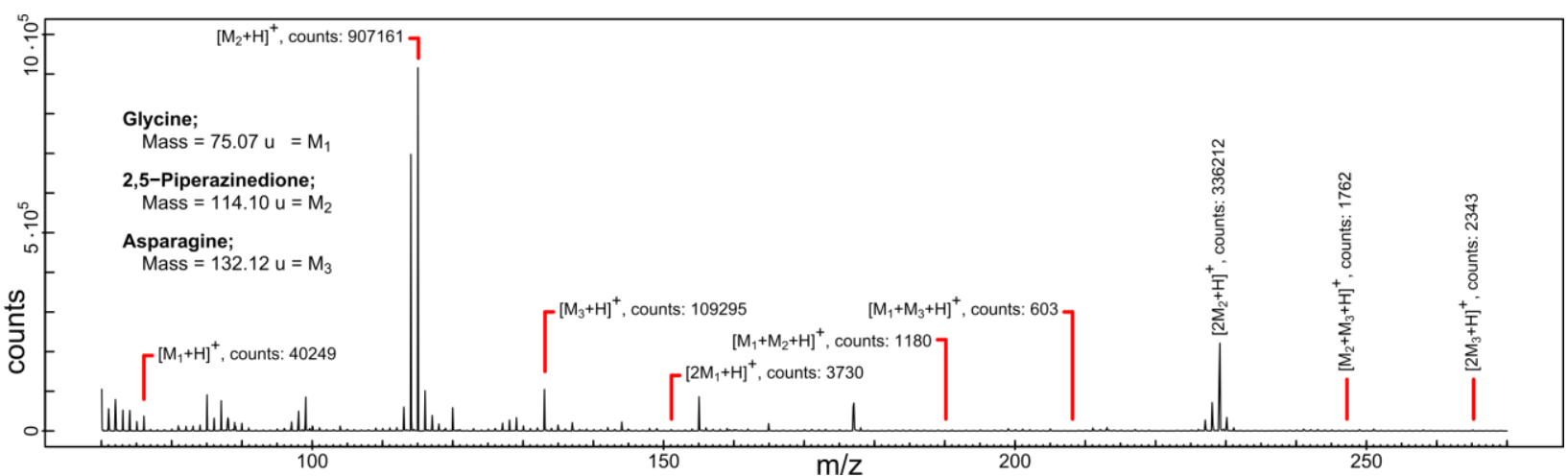

Fig. 3. Positive polarity spectrum acquired on a film consisting of glycine (Gly), 2,5piperazinedione (2,5-P.) and asparagine (Asp).

\begin{tabular}{|r|r|r|r|r|r|r|r|r|}
\hline$[\mathrm{Gly}+\mathrm{H}]^{+}$ & {$[[2,5-\mathrm{P} .]+\mathrm{H}]^{+}$} & {$[\mathrm{Asp}+\mathrm{H}]^{+}$} & {$[2 \mathrm{Gly}+\mathrm{H}]^{+}$} & {$[2[2,5-\mathrm{P} .]+\mathrm{H}]^{+}$} & {$[2 \mathrm{Asp}+\mathrm{H}]^{+}$} & {$[\mathrm{Gly}+[2,5-\mathrm{P} .]+\mathrm{H}]^{+}$} & {$[\mathrm{Gly}+\mathrm{Asp}+\mathrm{H}]^{+}$} & {$[[2,5-\mathrm{P} .]+\mathrm{Asp}+\mathrm{H}]^{+}$} \\
\hline 40249 & 907161 & 109295 & 3730 & 336212 & 2343 & 1180 & 603 & 1761 \\
\hline
\end{tabular}

Table 1. Total counts for monomer and dimer peaks in a positive spectrum acquired on a thin film consisting of glycine (Gly), 2,5-piperazinedione (2,5-P.) and asparagine (Asp). The counts were integrated over the full peak width. Note, the $[\mathrm{Gly}+\mathrm{Asp}+\mathrm{H}]^{+}$peak is below the noise floor as defined by the intensity of the surrounding $1 \mathrm{u}$ periodic peaks. In Fig. 4 the 
$[\mathrm{Gly}+\mathrm{Asp}+\mathrm{H}]^{+}$dimer formation probability is considered as zero for the bar plot computation and the integrated count rate (here red) of the peak occurring at the $[\mathrm{Gly}+\mathrm{Asp}+\mathrm{H}]^{+}$position is accounted for within the error bars in Fig. 4. 


\begin{tabular}{|l|r|l|l|}
\cline { 2 - 4 } \multicolumn{1}{c|}{} & Gly & $2,5-P$. & Asp \\
\hline Gly & $67.66 \%$ & $21.40 \%$ & $10.94 \%$ \\
\hline $2,5-P$. & $0.35 \%$ & $99.13 \%$ & $0.52 \%$ \\
\hline Asp & $12.81 \%$ & $37.42 \%$ & $49.77 \%$ \\
\hline
\end{tabular}

\begin{tabular}{|l|r|r|r|}
\cline { 2 - 4 } \multicolumn{1}{c|}{} & Gly & $2,5-P$. & Asp \\
\hline Gly & $89.78 \%$ & $2.41 \%$ & $7.81 \%$ \\
\hline $2,5-P$. & $0.66 \%$ & $98.42 \%$ & $0.92 \%$ \\
\hline Asp & $24.46 \%$ & $10.51 \%$ & $65.03 \%$ \\
\hline
\end{tabular}

\begin{tabular}{|l|r|r|r|}
\cline { 2 - 4 } \multicolumn{1}{c|}{} & \multicolumn{1}{c|}{ Gly } & 2,5-P. & \multicolumn{1}{l|}{ Asp } \\
\hline Gly & $75.97 \%$ & $24.03 \%$ & $0 \%$ \\
\hline $2,5-\mathrm{P}$. & $0.35 \%$ & $99.13 \%$ & $0.52 \%$ \\
\hline Asp & $0 \%$ & $42.92 \%$ & $57.08 \%$ \\
\hline
\end{tabular}

\begin{tabular}{|l|r|r|r|}
\cline { 2 - 4 } \multicolumn{1}{c|}{} & \multicolumn{1}{l|}{ Gly } & 2,5-P. & Asp \\
\hline Gly & $97.38 \%$ & $2.62 \%$ & $0 \%$ \\
\hline $2,5-\mathrm{P}$. & $0.66 \%$ & $98.42 \%$ & $0.92 \%$ \\
\hline Asp & $0 \%$ & $13.92 \%$ & $86.01 \%$ \\
\hline
\end{tabular}

Table 2. Interaction partner distributions for glycine (Gly), 2,5-piperazinedione (2,5-P.) and asparagine (Asp). (A,C) Interaction partner distributions as computed purely from the $\left[\mathrm{M}_{\mathrm{i}}+\mathrm{M}_{\mathrm{j}}+\mathrm{H}\right]^{+}$peak intensities by $\mathrm{X}_{\mathrm{ij}}^{\mathrm{i}}=100^{*}\left[\mathrm{M}_{\mathrm{i}}+\mathrm{M}_{\mathrm{j}}+\mathrm{H}\right]^{+} / \boldsymbol{\Sigma}_{\mathrm{n}}\left(\left[\mathrm{M}_{\mathrm{i}}+\mathrm{M}_{\mathrm{n}}+\mathrm{H}\right]^{+}\right)$, where $\mathrm{X}_{\mathrm{ij}}^{\mathrm{i}}$ is the estimated percentage of supramolecular dimers containing molecule $\mathrm{M}_{\mathrm{i}}$ that also contain molecule $\mathrm{M}_{\mathrm{j}}$. (B,D) Interaction partner distributions as computed taking molecular ionization potentials into account by means of Equation (7). Note, this normalization implicitly assumes that the ionization potential of a supramolecular dimer is a function of the ionization potentials of its components, however, the hydrogen bonds present in the dimer influence the ability of the dimer to accept a proton. (C,D) Fractional dimer compositions as computed after setting the $[\mathrm{Gly}+\mathrm{Asp}+\mathrm{H}]^{+}$peak intensity to zero.

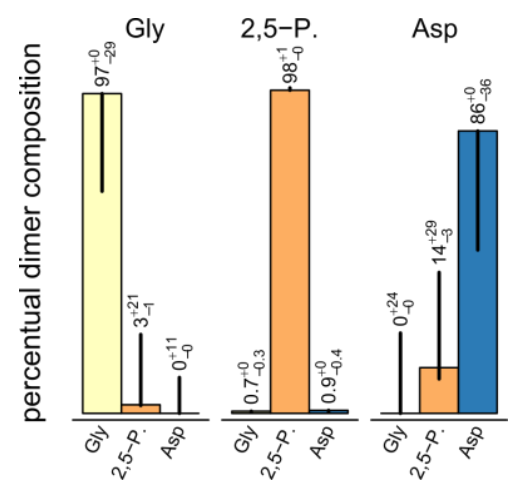

Fig. 4. Molecular interactions in a thin film containing glycine, 2,5-piperazinedione and

asparagine. Relative probabilities to form a non-covalent pair with a specific pairing partner for each of the molecules in a drop-deposited film consisting of glycine (Gly), asparagine (Asp) and 2,5-piperazinedione (2,5-P.) in the molar ratio 1:1:1. The values plotted are computed through normalization of the $\left[\mathrm{M}_{\mathrm{i}}+\mathrm{M}_{\mathrm{j}}+\mathrm{H}\right]^{+}$counts to the ionization potential of its constituting molecules as obtained from the $\left[\mathrm{M}_{\mathrm{i} / \mathrm{j}}\right]^{+}$counts. The error bars indicate the variation of each value when computed with the ionization potential unaccounted for or when no assumption is made concerning the noise content of the $[\mathrm{Gly}+\mathrm{Asp}+\mathrm{H}]^{+}$peak (see Table 2, panels A-C). The extraction, transmission and detection probabilities are assumed equal for all pairings. 


\section{Conclusions}

We demonstrated that, under a broad range of conditions, supramolecular assemblies and networks are commonly preserved throughout the processes of desorption and ionization. Such observation paves the way to use SIMS as a unique tool in the study of molecular interactions and short-range supramolecular ordering in non-crystalline organic matter. Mass spectrometry is already successfully used in the identification of the structure of complex branched organic macromolecules (22), and we suggest analogue procedures for the analysis of supramolecular structuring. Similarly, procedures that have been originally developed for ESI $(3,5)$ can be adapted in the estimation of probabilities for the occurrence of non-covalent bonding between specific molecules in organic and biological solid and soft matter. The presented results demonstrate that non-covalent interactions can be probed at the scale of single molecules, revealing aggregation and self-assembly not accessible to electron microscopy or X-ray diffraction.

\section{References and Notes:}

1. C. H. Schalley, Int. J. Mass. Spectrom., 2000, 194, 11-39

2. T. Wyttenbach and M. T. Bowers, Annu. Rev. Phys. Chem., 2007, 58, 511-588.

3. S. M. Clark and L. Konermann, Anal. Chem., 2004, 76, 1257-1263.

4. J. A. Loo, Mass Spectrom. Rev., 1997, 16, 1-23.

5. E. B. Erba, K. Barylyuk, Y. Yang and R. Zenobi, Anal. Chem., 2011, 83, 9251-9259.

6. D. S. Chorev, L. A. Baker, D. Wu, V. Beilsten-Edmands, S. L. Rouse, T. Zeev-BenMordehai, C. Jiko, F. Samsudin, C. Gerle, S. Khalid, A. G. Stewart, S. J. Matthews, K. Grünewald and C. V. Robinson, Science, 2019, 362, 829-834.

7. S. Mädler, E. B. Erba and R. Zenobi, in Applications of MALDI-ToF Spectrometry, 2013, pp $1-36$.

8. H. T. Jonkman and J. Michl, in Secondary Ion Mass Spectrometry SIMS II, 1979, pp. 292295.

9. R. G. Orth, H. T. Jonkman and J. Michl, J. Am. Chem. Soc., 1981, 103, 1564-1565.

10. F. Kollmer, Appl. Surf. Sci., 2004, 231-232, 153-158.

11. D. Touboul, F. Kollmer, E. Niehuis, A. Brunelle and O. Laprévote, J. Am. Soc. Mass Spectrom., 2005, 16, 1608-1618.

12. M. Kawecki and L. Bernard, Surf. Sci. Spectra, 2018, 25, 015001

13. M. Kawecki and L. Bernard, Surf. Sci. Spectra, 2018, 25, 015002

14. L. Bernard, R. Crockett, and M. Kawecki, Surf. Sci. Spectra, 2019, 26, 025001

15. L. Bernard, R. Crockett, and M. Kawecki, Surf. Sci. Spectra, 2019, 26, 025002

16. R. Peverati and D. G. Truhlar, J. Phys. Chem. Lett., 2011, 2, 2810-2817.

17. S. Myung, K. P. Lorton, S. I. Merenbloom, M. Fioroni, S. L. Koeniger, R. R. Julian, M.-H. Baik and D. E. Clemmer, J. Am. Chem. Soc., 2006, 128, 15988-15989. 
18. R. B. Corey, J. Am. Chem. Soc., 1938, 60, 1598-1604.

19. R. Degeilh and R. E. Marsh, Acta Cryst., 1959, 12, 1007-1014.

20. J. Huddleston, A. Veide, K. Köhler, J. Flanagan, S.-O. Enfors and A. Lyddiatt, Trends Biotechnol., 1991, 9, 381-388.

21. K. Ratanapongleka, Int. J. Chem. Eng. Appl., 2010, 1, 191-198.

22. A. S. Weiskopf, P. Vouros and D. J. Harvey, Anal. Chem., 1998, 70, 4441-4447.

23. M. W. Schmidt, K. K. Baldridge, J. A. Boatz, S. T. Elbert, M. S. Gordon, J. H. Jensen, S. Koseki, N. Matsunaga, K. A. Nguyen, S. Su, T. L. Windus, M. Dupuis and J. A. Montgomery, J. Comput. Chem., 1993, 14, 1347-1363.

24. S. F. Boys and F. Bernardi, Mol. Phys., 1970, 19, 553-566.

25. E. F. Pettersen, T. D. Goddard, C. C. Huang, G. S. Couch, D. M. Greenblatt, E. C. Meng and T. E. Ferrin, J. Comput. Chem., 2004, 25, 1605-1612.

26. B. M. Bode and M. S. J. Gordon, J. Mol. Graph. Model., 1999, 16, 133-138.

27. R. G. Cooks, D. Zhang and K. J. Koch, Anal. Chem., 2001, 73, 3646-3655

Conflicts of interest: There are no conflicts of interest to declare. Acknowledgments: Dr. Andres Kaech (University of Zürich), Prof. Karl-Heinz Ernst (University of Zürich, Empa), Prof. Hans J. Hug (University of Basel, Empa), Dr. Delphine Kawecki-Wenger (ETH Zürich, Empa) and Prof. Jess Snedeker (ETH Zürich, University clinic Balgrist) are thanked for fruitful discussions. Funding: The work performed at Empa Dübendorf was supported by the Swiss National Science Foundation grant number CR23I2-162828. Author contributions: M.K. and L.B. observed systematic supramolecular secondary ion formation. M.K. conceived, designed and directed the study; performed and analyzed the experiments. D.P. conducted the quantum chemistry simulations. R.C. advised sample chemistries. M.K. wrote the manuscript which was reviewed by all authors. Data availability: The raw data that support the findings of this study are available from the corresponding author upon request and a copy is backed up on the data server of Empa - Swiss Federal Laboratories for Materials Science and Technology. 


\section{Supplementary Materials:}

\section{Materials and Methods}

Silicon substrate preparation. $1 \times 1 \mathrm{~cm}^{2}$ silicon wafers were cleaned in three steps in an ultrasound bath over a duration of 2x15 min in Acetone and 1x15 min in ethanol and subsequently exposed to oxygen plasma over a duration of $10 \mathrm{~min}$.

Sample preparation. Sample molecules were purchased in high purity from Sigma Aldrich, Dextran (T5, 5.2 kDa, polydispersity 1.8) from Pharma-cosmos A/S, Denmark. The molecules were separately dissolved in pure $\mathrm{H}_{2} \mathrm{O}$ (Sigma-34877) at a concentration of $3 \mu \mathrm{g} / \mathrm{ml}$ and agitated using a vortex mixer to prepare pure stock solutions. Out of these stock solutions, sample solutions were prepared by pipetting to achieve the mass ratios indicated in the manuscript and again agitated using a vortex mixer. $50 \mu 1$ of each sample was drop-deposited on a separate plasmatreated silicon wafer of $1 \times 1 \mathrm{~cm}^{2}$. It was assured that the deposited droplet spread over the entire available Si wafer surface, which had been rendered hydrophilic by the plasma treatment minutes prior to drop deposition. The samples were left to dry in a laminar flow chamber and analyzed the same day by ToF-SIMS.

15 ToF-SIMS data acquisition. Secondary ion mass spectrometry measurements were conducted on a ToF-SIMS.5 from IONTOF GmbH, Germany. The instrument was operated in spectroscopy mode and $\mathrm{Bi}_{3}{ }^{+}$primary ions were used to analyze an area of $100 \times 100 \mu \mathrm{m}^{2}$. The extractor voltage used was $3000 \mathrm{~V}$, providing increased sensitivity in the high mass range.

Quantum chemistry simulations. All quantum chemistry calculations were performed using the GAMESS-US package (23) using the 6-311(2d,p) basis sets for all elements. The geometries were fully optimized up to a force threshold of $10^{-4}$ a.u. and the binding energy computed including the basis set superposition error correction using the procedure by Boys and Bernardi (24). We conducted all calculations with several exchange-correlation functionals of the meta-GGA family (16 and references therein), finding minimal differences in the binding energies. The reported binding energies and configurations were obtained with the M11 functional, a rangeseparated hybrid functional with $42.8 \%$ Hartree-Fock exchange in the short-range and $100 \%$ in the long-range. The figures including the molecular electrostatic potential coloring of the density isosurface (Fig. $1 \mathrm{C}$ ) are realized with the software UCSF Chimera (25) interfaced with the package MacMolPIt (26). 


\section{Supplementary Materials:}

Selected further examples of supramolecular secondary ions in ToF-SIMS mass spectra acquired on drop-deposited films

Fig. S1

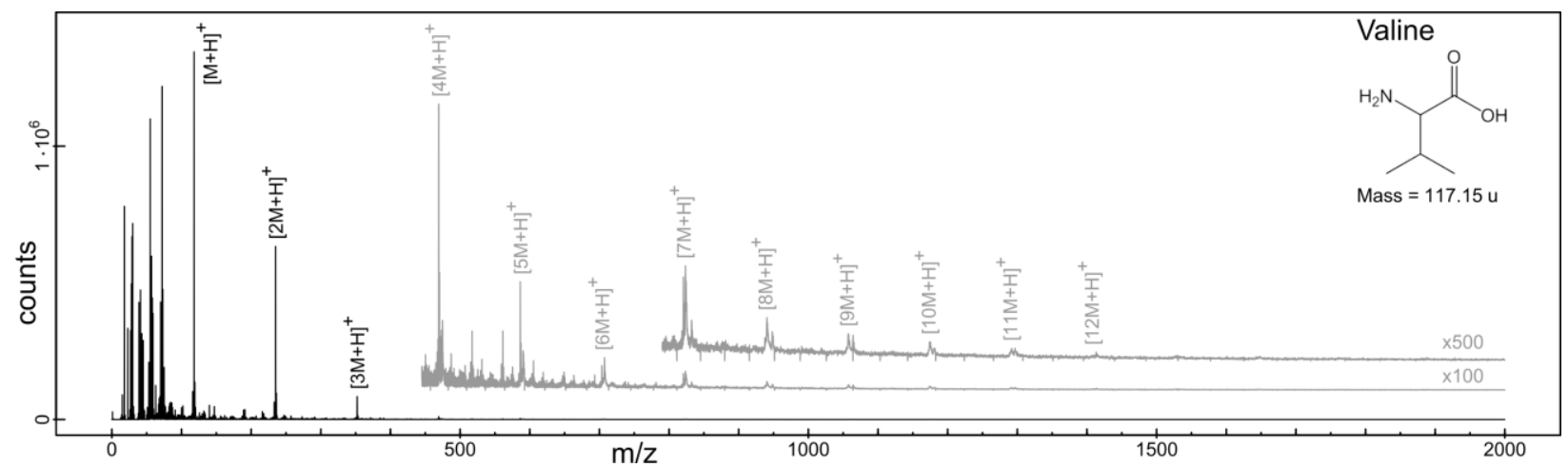

Positive ToF-SIMS spectrum of the amino acid L-valine. 
Fig. S2

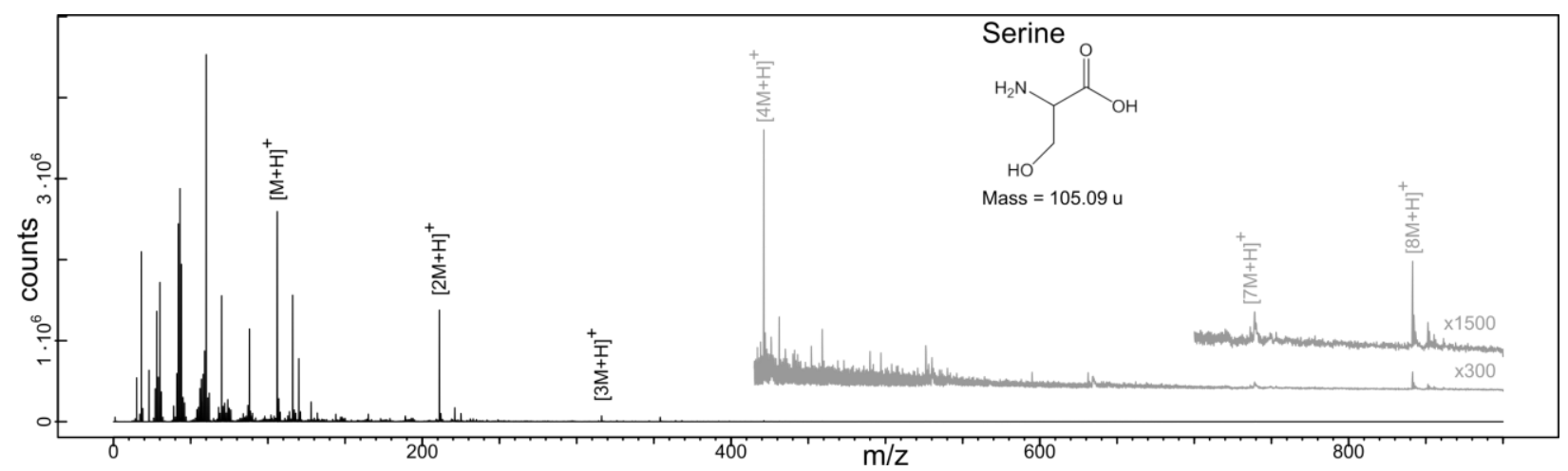

Positive ToF-SIMS spectrum of the amino acid L-serine. Note the distinct peak at mass $[8 \mathrm{M}+\mathrm{H}]^{+}$which has a higher intensity than the $[6 \mathrm{M}+\mathrm{H}]^{+}$and the $[7 \mathrm{M}+\mathrm{H}]^{+}$peaks. Serine was in the past observed to form stable, homochiral octameric clusters upon electrospray ionization of serine-containing solutions (27). The extraction of octameric serine clusters as supramolecular secondary ions by ToF-SIMS indicates that this arrangement occurs also upon solidification of serine on solid surfaces. 
Fig. S3

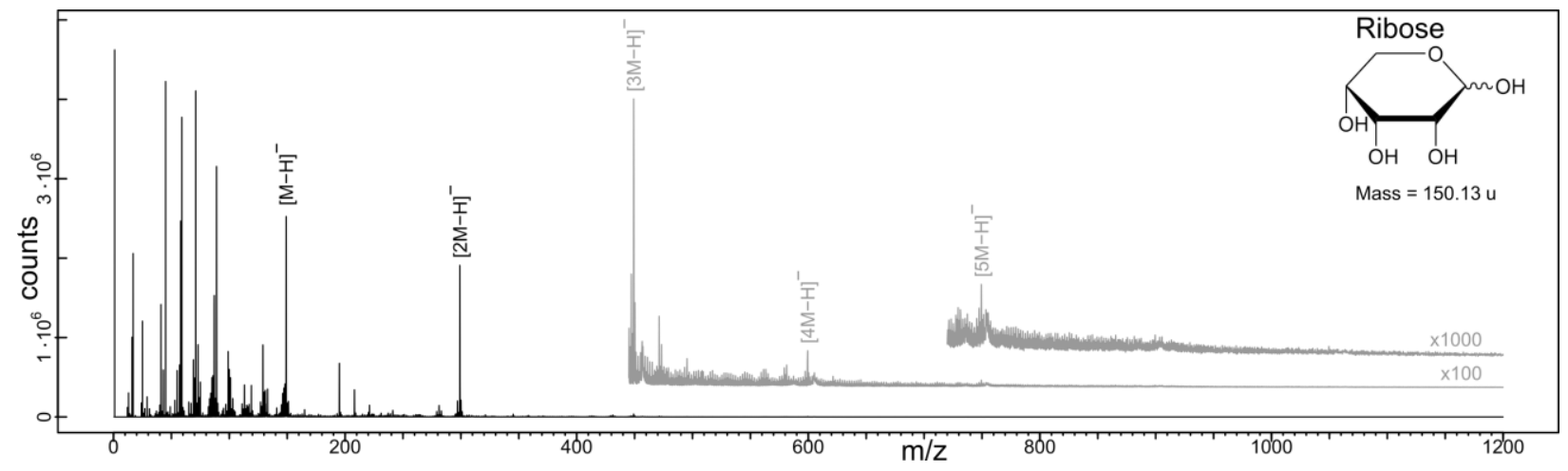

Negative ToF-SIMS spectrum of the monosaccharide D-ribose. Note that monosaccharides preferentially ionize

by loss of a proton $\left(\mathrm{H}^{+}\right)$. Correspondingly, most supramolecular secondary ions extracted are negatively charged and have the form $[\mathrm{nM}-\mathrm{H}]^{-}$. 
Fig. S4

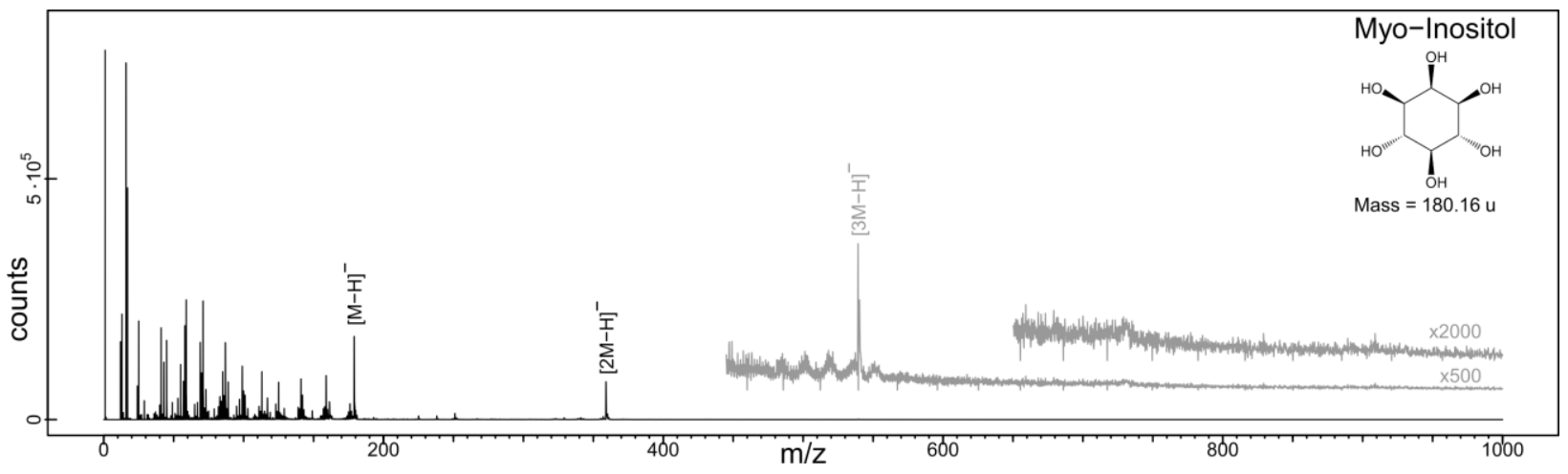

Negative ToF-SIMS spectrum of the monosaccharide myo-inositol. 
Fig. S5

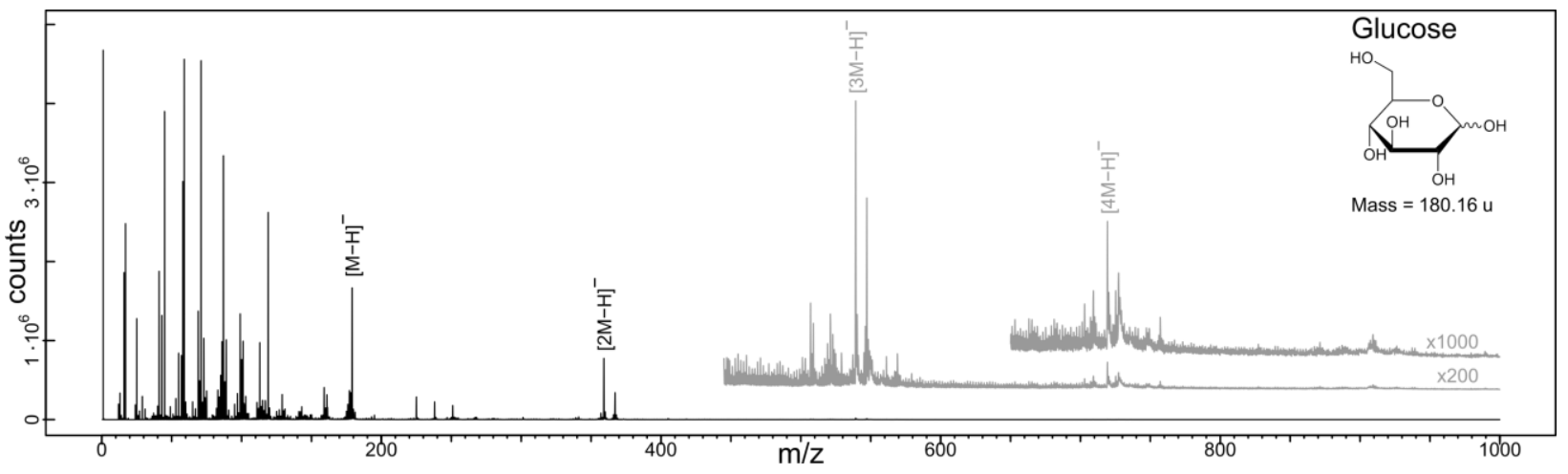

Negative ToF-SIMS spectrum of the monosaccharide D+glucose. 